\title{
Determinación rápida de la cal libre: Modificación de los métodos Franke y Schlaepfer-Bukowski
}

\author{
F. MASSAZZA Y M. T. FRANCARDI \\ II Cemento, n० 2, febrero 1970, págs. 55-60
}

\section{INTRODUCCION}

La determinación cuantitativa de la cal libre en el clínker constituye un control industrial de primordial importancia, por cuanto representa un índice de la regularidad del proceso de producción del clínker.

Esta determinación se lleva a cabo, en la mayoría de los casos con procedimientos químicos basados en la extracción selectiva del óxido de calcio $\left({ }^{*}\right)$ y en la subsiguiente valoración.

Los procedimientos analíticos más destacados y actualmente empleados en mayor escala son: el método Franke (1) y el método Schlaepfer-Bukowski (2), eventualmente modificado por S. Rordam (3) y Bielatowicz (4).

Se ha escrito mucho acerca de las ventajas y limitaciones de ambos métodos (5), (6), (7), (8) y (9), pero se sale de la finalidad del presente trabajo recoger estos argumentos. Es de recordar que el método Franke es quizás el más empleado, a pesar de tener la desventaja de requerir un tiempo de análisis superior en 30 minutos al método SchlaepferBukowski. En favor del primer método se encuentra la menor influencia de la finura de la muestra, la extracción casi omisible de los álcalis y del magnesio y el viraje muy neto del indicador de $\mathrm{pH}$.

El método Schlaepfer-Bukowski encuentra todavía mucha aplicación por su mayor rapidez y también por el menor costo de los reactivos.

Como conclusión, el método Franke proporciona mayores garantías de precisión y reproducibilidad, pero resulta más bien lento.

Este inconveniente, omisible en otras circunstancias, puede representar una grave ventaja cuando la determinación de cal libre constituye el control de base para la comprobación de la calidad de una producción continua y masiva. En estos casos es en realidad conveniente, casi indispensable, que la información necesaria se adquiera cuanto más rápidamente sea posible para permitir las oportunas intervenciones correctoras sobre el proceso tecnológico.

Por eso, para poder disponer de un método de análisis lo más rápido posible, se recogen en este examen los dos métodos antes citados, modificándolos oportunamente para adaptarlos mejor a las exigencias del control de una producción industrial.

(*) Estos métodos dosifican junto al $\mathrm{CaO}$ el $\mathrm{Ca}(\mathrm{OH})_{2}$ eventualmente presente. 
En otros términos, se ha tratado de realizar un método Franke más rápido y un método Schlaepfer-Bukowski más preciso, con objeto de suministrar, en cualquier caso, resultados analíticos prácticamente coincidentes con los obtenibles mediante el método original de Franke.

Los ensayos se han hecho sobre 30 muestras de clínker, procedentes de otras tantas fábricas de cemento pertenecientes al grupo Italcementi. Las muestras, todas diferentes, tanto por el origen como por la composición química y potencial, se han molido hasta una finura Blaine de $3.500 \mathrm{~cm}^{2} / \mathrm{g}$. La muestra n. ${ }^{\circ} 4$ representa un caso provocado voluntariamente de un clínker con elevado contenido de cal libre.

\section{PARTE EXPERIMENTAL}

\subsection{Método Franke modificado}

\subsubsection{POSIBLES MODIFICACIONES DEL METODO}

Para acelerar el procedimiento analítico de este método es indispensable poder abreviar el proceso de extracción que en él constituye la operación más larga (60 minutos); pero las pruebas realizadas en este sentido han confirmado que, especialmente en el caso de muestras de elevado contenido de cal libre, se obtienen sistemáticamente resultados inferiores al previsto.

Puesto que no resulta conveniente aplicar la simple reducción del tiempo de extracción, se ha estudiado un catalizador que, manteniendo inalteradas las características de precisión propias del método Franke original, reduce drásticamente el tiempo de extracción.

Esta investigación se basa en la siguiente consideración: estando asegurado que en el método Franke es la forma enólica del éter la que reacciona con la cal libre (1), un catalizador capaz de desplazar el equilibrio hacia la formación de la forma enólica aceleraría la reacción de salificación con el óxido de calcio.

La elección del catalizador recayó sobre el acetato de mercurio, $\mathrm{HgAc}_{2}$, que pertenece a un grupo de compuestos, sales de óxidos metálicos en su mayor parte, que catalizan precisamente las reacciones orgánicas del tipo de la transeterificación y enolización (10).

La acción catalítica no tendrá lugar directamente a partir del acetato de mercurio, sino de su producto de hidrólisis $\mathrm{HgO}$, y por lo tanto, es necesario añadir a la sal una cantidad limitada de agua.

\subsubsection{RESULTADOS}

Con el fin de comprobar la presunta acción del acetato de mercurio se ha modificado el reactivo de Franke, sustituyendo el alcohol isobutílico puro, por alcohol isobutílico que contiene $0,1 \%$ de $\mathrm{HgAc}_{2}$ y una pequeña cantidad de agua $(0,1 \%)$ necesaria para favorecer la hidrólisis del acetato (reactivo A).

El catalizador se ha empleado en dos series de ensayo, en las cuales los tiempos de extracción han sido de 15 y 30 minutos, respectivamente. Los resultados se encuentran en la tabla 1 (columnas $3 .^{a}$ y $4 .{ }^{a}$ ) y en ella se confrontan con los obtenidos con el método Franke, tanto normal (columna $1 .^{a}$ ) como con tiempo de extracción reducido a 15 minutos (columna 2. ${ }^{\mathrm{a}}$ ). 


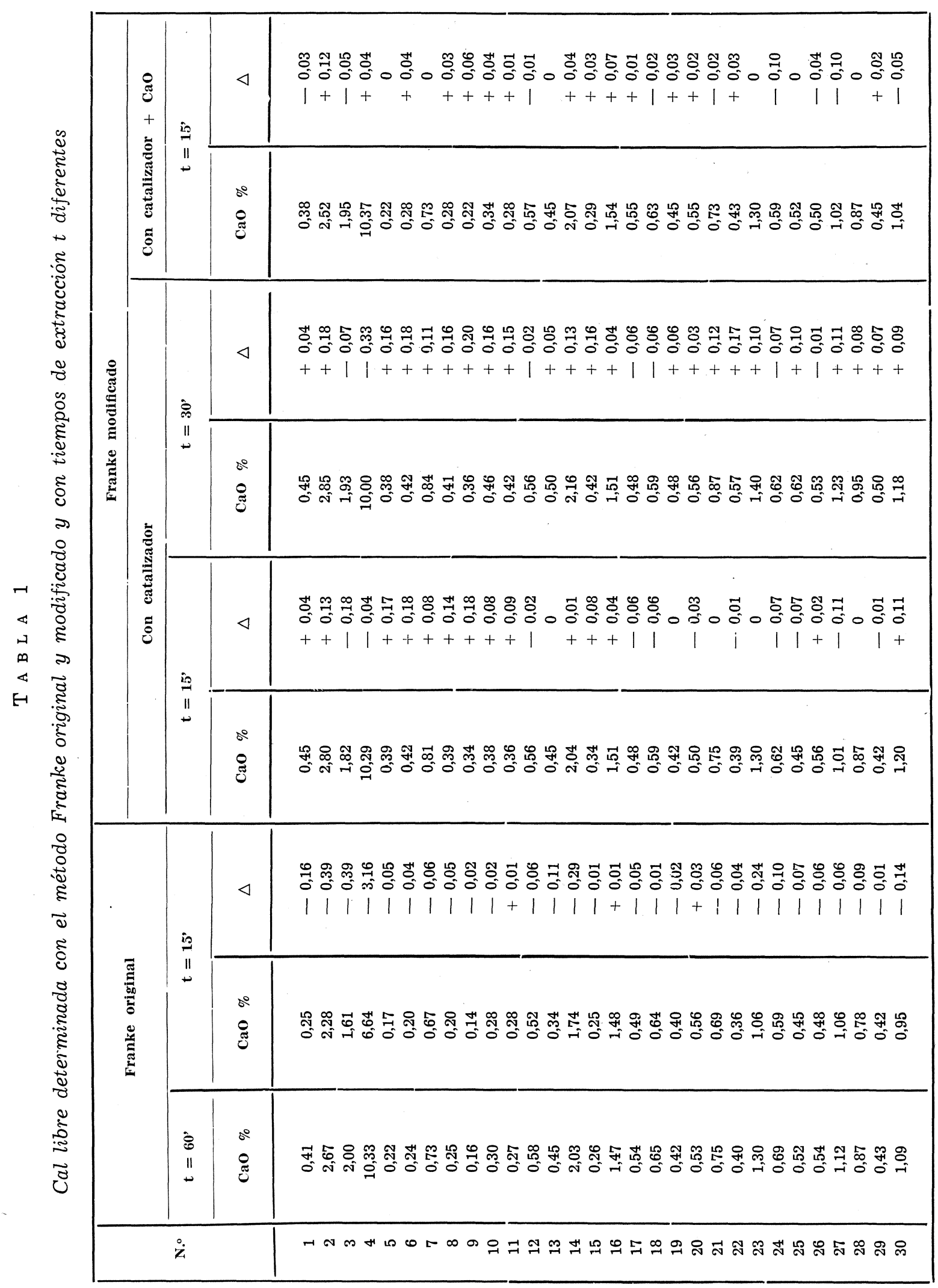


En la tabla 1 se observa que la reducción del tiempo de extracción (sin catalizador) a 15 minutos lleva consigo - casi sistemáticamente- errores en defecto, que pueden hacerse graves cuando el contenido en cal libre es elevado.

Empleando el reactivo A, es decir, el agente catalizador, los resultados aparecen generalmente en ligero exceso. En cualquier caso, 15 minutos son suficientes para completar el proceso de extracción.

Suponiendo que tales inconvenientes puedan atribuirse a alguna acción agresiva inicial del reactivo A sobre los otros constituyentes del cemento, el éter acetacético puro se ha sustituido por una solución de éter acetacético en alcohol isobutílico conteniendo una pequeña cantidad de $\mathrm{CaO}$ (reactivo B).

Aplicando juntos estos dos refinamientos, es decir, empleando el acetato de mercurio como catalizador y el óxido de calcio como moderador de la agresividad inicial del reactivo de extracción, se han obtenido resultados experimentales (columna 5.a) que, además de estar en completo acuerdo con el método Franke original, presentan la importante ventaja de requerir un tiempo de extracción muy reducido: 15 contra 60 minutos.

\subsubsection{DESCRIPCION DEL METODO FRANKE MODIFICADO}

\subsubsection{Reactivos necesarios}

- alcohol metílico;

- alcohol isobutílico;

- éter acetacético;

- ácido clorhídrico, solución acuosa $0,1 \mathrm{~N}$;

- azul de bromofenol, solución acuosa al $1 \%$;

- acetato mercúrico, $\mathrm{HgAc}_{2}$;

- carbonato cálcico;

- reactivo A: alcohol isobutílico conteniendo el $0,1 \%$ de $\mathrm{HgAc}_{2}$ y de agua.

(Preparación: Se pesa un gramo de $\mathrm{HgAc}_{2}$ y se coloca en un matraz de 1.000 c.c.; se añade 1 c.c. de agua y se completa el volumen con alcohol isobutílico. Se debe agitar sobre placa magnética alrededor de 30 minutos para facilitar la hidrólisis del $\mathrm{HgAc}_{2}$. Se obtiene así una suspensión de $\mathrm{HgO}$ en el medio alcohólico, por lo que es aconsejable agitar cada vez que se tome una fracción).

- reactivo $\mathrm{B}$ : alcohol isobutílico conteniendo $0,02 \%$ de $\mathrm{CaO}$ y $30 \%$ de éter acetacético.

(Preparación: Se hierven a reflujo, durante una hora 40 c.c. de alcohol isobutílico, 6 c.c. de éter acetacético, adicionados de $0,2 \mathrm{~g}$ de $\mathrm{CaO}$, obtenido por calcinación a $950^{\circ} \mathrm{C}$ de $\mathrm{CO}_{3} \mathrm{Ca}$ puro; se enfría, se trasvasa cuantitativamente a un matraz aforado de 1.000 c.c., se añaden 300 c.c. de éter acetacético y se completa el volumen con alcohol isobutílico. Más tarde se determina el título en cal valorando 10 c.c. con una solución de $\mathrm{ClH} 0,1 \mathrm{~N}$ acuosa o alcohólica).

\subsubsection{Técnica experimental}

El aparato de reacción está constituido por un matraz de 150 c.c., con cono esmerilado, provisto de un refrigerante de bolas, con terminal de cloruro cálcico y cal sodada. 
Se trabaja sobre un gramo de muestra a la que se añaden 15 c.c. del reactivo A medidos con una probeta graduada corriente y 10 c.c. del reactivo $B$, tomados con una pipeta de precisión.

Se lleva a ebullición sobre agitador magnético con placa calefactora, manteniéndolo con enérgica agitación durante 15 minutos.

Se filtra sobre Buckner, con dos filtros de banda azul empleando un matraz de vacío de 250 c.c.; se lava de 2 a 3 veces con alcohol isobutílico y se trasvasa el filtrado cuantitativamente a un matraz de 250 c.c.

Se añaden al filtrado 20 c.c. de alcohol metílico y se valora con una solución de $\mathrm{ClH} 0,1$ $\mathrm{N}$ con el indicador de azul de bromofenol.

\subsubsection{Cálculo de los resultados}

Para calcular el porcentaje de $\mathrm{CaO}$, se recurre a la siguiente expresión:

$$
\% \text { de cal libre }=\left(\mathrm{V}_{\mathrm{x}}-\mathrm{V}_{1}\right) 0,2804 ;
$$

donde:

$\mathrm{V}_{\mathrm{x}}=$ volumen de $\mathrm{ClH} 0,1 \mathrm{~N}$ consumido en la valoración;

$\mathrm{V}_{1}=$ volumen de $\mathrm{ClH} 0,1 \mathrm{~N}$ consumido al valorar 10 c.c. del reactivo $\mathrm{B}$.

\subsection{Método Schlaepfer-Bukowski modificado}

\subsubsection{POSIBLES MODIFICACIONES DEL METODO}

Es sabido que el método Schlaepfer-Bukowski en su versión original, proporciona, respecto al método Franke, resultados más rápidos, pero sistemáticamente en exceso. Esto depende en parte del hecho de que el etilenglicol extrae, no sólo la cal libre, sino también parte de los álcalis contenidos en la muestra, álcalis que durante la valoración acidimétrica se dosifican inevitablemente junto con la cal.

Este inconveniente se elimina sólo parciaimente sustituyendo la valoración acidimétrica por la valoración complexométrica de la cal y además, a pesar de esta mejoría, el método sigue dando resultados en exceso. En cualquier caso, el empleo del método complexométrico representa una indudable ventaja, porque permite eliminar la interferencia no sólo de los álcalis, sino también de la pequeña cantidad de magnesio presente en el extracto.

\subsubsection{RESULTADOS}

En el intento de eliminar las diferencias con el método Franke original, empleado como método de referencia, y de acelerar posteriormente el procedimiento analítico, se ha considerado el estudio de la influencia ejercida por la disminución del tiempo de extracción sobre los resultados obtenibles con el método Schlaepfer-Bukowski, modificado sólo en lo que respecta a la valoración complexométrica de la cal libre.

Los ensayos se han hecho sobre las 15 muestras de clínker examinadas anteriormente.

Observando la tabla 2 (columnas $1 .^{a}, 2 .^{a}, 3 .^{a}$ y $4 .{ }^{a}$ ) está claro que, aun habiendo eliminado la interferencia de los álcalis y del magnesio, los resultados son todavía muy elevados, incluso limitando el proceso de extracción a sólo 5 minutos. 


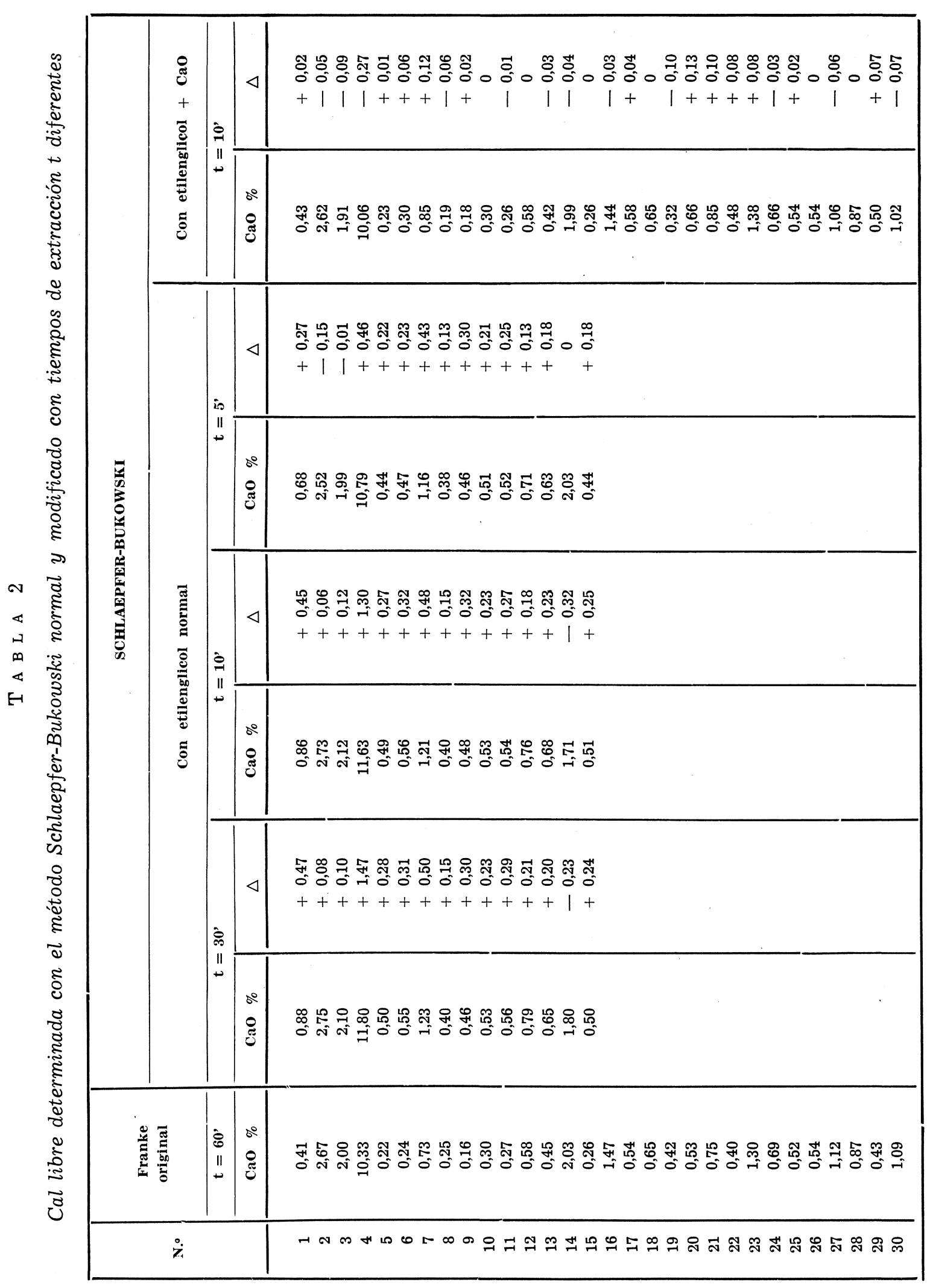


La sistematicidad de estos errores "en exceso" que, particularmente interesante, depende poco del tiempo de extracción, ha sugerido la hipótesis de que el etilenglicol pueda ejercer inicialmente una acción agresiva generalizada ( $y$, por tanto, no limitada sólo a la cal libre) que, en un segundo tiempo, con el paso de la cal a la solución, desaparezca.

Para bloquear este ataque sobre los demás constituyentes, el etilenglicol puro se ha sustituido con glicol, el cual contiene ya en parte una pequeña cantidad de $\mathrm{CaO}(0,2 \mathrm{~g}$ en un litro).

Este simple refinamiento, como en el caso del método de Franke, ha mostrado ser eficacísimo, limitando a sólo 10 minutos la extracción con el disolvente propuesto. Además, la concordancia entre los resultados obtenidos con el método Schlaepfer-Bukowski modificado y el método Franke original, tomado como referencia (ver columnas $1 .^{\mathrm{a}}$ y $5 .^{\mathrm{a}}$ de la tabla 2), ha resultado óptima en los 30 casos estudiados.

Las modificaciones aportadas a este método presentan la ventaja, tanto de eliminar el ataque a los constituyentes mineralógicos distintos del $\mathrm{CaO}$, como de reducir el tiempo de análisis completo a menos de media hora.

\subsubsection{DESCRIPCION DEL METODO SCHLAEPFER-BUKOWSKI MODIFICADO}

\subsubsection{Reactivos necesarios}

- solución 0,03 M de EDTA;

- solución acuosa de $\mathrm{NaOH}$ al $10 \%$;

- alcohol metílico;

— indicador Murexida (mezcla seca al $1 \%$ en ClK molido);

- solución de $\mathrm{CaO}$ en etilenglicol: se prepara agitando prolongadamente en un litro de glicol $0,2 \mathrm{~g}$ de $\mathrm{CaO}$, preparado calcinando a $950^{\circ} \mathrm{C}$ el $\mathrm{CO}_{3} \mathrm{Ca}$ puro.

\subsubsection{Técnica experimental}

Se pesa un gramo de muestra (clínker finamente molido, sin residuo en el tamiz de 4.900 mallas $/ \mathrm{cm}^{2}$ ) y se introduce en un matraz pyrex de 200 c.c., perfectamente seco.

Se introducen 50 c.c. exactos de la solución de $\mathrm{CaO}$ en etilenglicol, se incorpora un agitador magnético revestido de teflon y se cierra el matraz con tapón de goma atravesado por un termómetro.

Se coloca sobre el agitador magnético con placa calefactora, cuidando alcanzar rápidamente, pero sin superarla, la temperatura de $68^{\circ}-70^{\circ} \mathrm{C}$.

Al cabo de 10 minutos, se filtra la suspensión a través de un Buchner con filtro seco de banda azul, recogiendo el filtrado en un matraz de vacío de 250 c.c. Se lava el matraz dos veces con unos 10 c.c. de etilenglicol puro, y finalmente se hace un lavado con 20 c.c. de alcohol metilico.

Se transfiere el filtrado a un vaso de 400 c.c., lavando esmeradamente el matraz con agua destilada, se añaden 40 c.c. de la solución de $\mathrm{NaOH}$ (el pH deberá ser de 12 a 12,5) y se valora con EDTA, empleando el indicador Murexida.

\subsubsection{Cálculo de los resultados}

$$
\% \text { de cal libre }=(\mathrm{T}-\mathrm{B}) \mathrm{f} \cdot 100 ;
$$


en la que:

T es volumen (en c.c.) de EDTA consumido en la valoración de la muestra en estudio;

B, el volumen (en c.c.) de EDTA consumido en la valoración de 50 c.c. de la solución de $\mathrm{CaO}$ en glicol;

f, el equivalente en $\mathrm{CaO}$ de 1 c.c. de EDTA.

\section{CONCLUSIONES}

Los ensayos anteriores han demostrado que modificando oportunamente los métodos de Franke y de Schlaepfer-Bukowski es posible abreviar notablemente los tiempos de análisis: los tiempos de extracción de la cal se reducen así, respectivamente, a 15 y $10 \mathrm{mi}$ nutos. En cuanto se refiere a los resultados analíticos, concuerdan perfectamente con los obtenidos aplicando el método original de Franke.

\section{R E S U M E N}

Modificando los métodos de Franke y Schlaepfer-Bukowski, es posible reducir notablemente el tiempo necesario para determinar la cal libre en los clínkeres y en los cementos.

Las modificaciones aportadas al método de Franke consisten en el empleo de un catalizador (acetato de mercurio) en el proceso de extracción, y en la sustitución del éter acetacético puro por una solución de éter acetacético en alcohol isobutílico conteniendo una pequeña cantidad de óxido cálcico.

Puesto que el método de Schlaepfer-Bukowski tiende a dar valores en exceso, que tienen su mayor justificación en un ataque inicial del etilenglicol sobre los demás componentes del clínker, el reactivo puro se ha sustituido por glicol conteniendo una pequeña cantidad de óxido de calcio.

Los dos métodos modificados aseguran resultados que están en perfecto acuerdo con los obtenidos aplicando el método original Franke y presentan la ventaja de reducir los tiempos de extracción a 5 y 10 minutos, respectivamente.

\section{R E F E R E N C I A S}

(1)

B. Franke: Z. Anorg. Allg. Chem., 247, 180, (1941).

P. SCHLAEPfeR, R. Bukowski: Eidgenössische Materialprüfungsanstalt an der E.T.H., Zürich, 63, (1933).

S. RORDAN: Rock Prod., 40, 72 (1957).

J. Bielatowicz: Cement-Wapno-Gips 18-28, 214 (1963).

A. Dubuisson: Rev. Mater. Constr. n. 398, 293, (1948).

G. O. Assarson, J. M. Bokstrom: Anal. Chem. 25, 1844 (1953).

G. Assarson: Zem. Kalk Gips 7, 167 (1954).

G. E. Pressler, S. Brunauder, D. L. Kantro: Anal. Chem. 28, 896, (1956).

L. Gloor, A. Bussmann: Bulletin Holderbank n. 155.

W. H. Watanabe, L. E. Conlon: J. Amer. Chem. Soc. 79, 2828, (1957). 